\title{
Closure Polynomials for Strips of Tetrahedra
}

Federico Thomas and Josep M. Porta

\begin{abstract}
A tetrahedral strip is a tetrahedron-tetrahedron truss where any tetrahedron has two neighbors except those in the extremes which have only one. Unless any of the tetrahedra degenerate, such a truss is rigid. In this case, if the distance between the strip endpoints is imposed, any rod length in the truss is constrained by all the others to attain discrete values. In this paper, it is shown how to characterize these values as the roots of a closure polynomial whose derivation requires surprisingly no other tools than elementary algebraic manipulations. As an application of this result, the forward kinematics of two parallel platforms with closure polynomials of degree 16 and 12 is straightforwardly solved.
\end{abstract}

Key words: Position analysis, closed-form solutions, Distance Geometry, spatial linkages.

\section{Introduction}

Let us consider the strip of tetrahedra in Fig. 1. Any such strip has two endpoints. In this case, $P_{a}$ and $P_{b}$. If the distance between these two points is imposed, the length of any rod cannot be freely chosen. This paper is essentially devoted to obtain a closed-form solution for the length of any rod in a strip of tetrahedra, once the distance between its endpoints and the lengths of all other rods are known.

Although closure polynomials have been typically obtained on a case-by-case analysis, a common pattern can be identified for most cases. First, a set of loop equations involving both translation and orientation variables is derived. Then, translation variables are eliminated resulting in a system of trigonometric equations that is algebraized using the tangent half-angle substitution. Finally, elimination theory is

Federico Thomas · Josep M. Porta

Institut de Robòtica i Informàtica Industrial, CSIC-UPC, Barcelona, Spain

e-mail: \{fthomas,porta\}@iri.upc.edu 


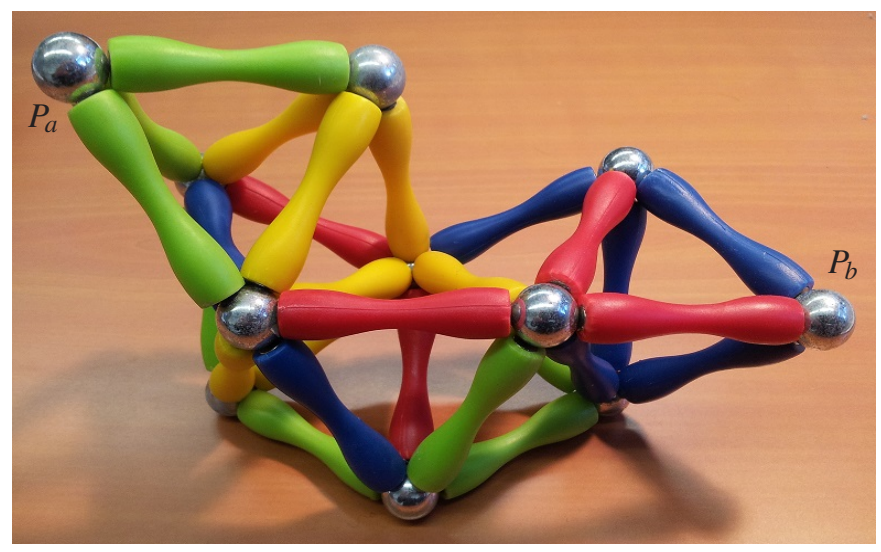

Fig. 1 A strip of eight tetrahedra whose endpoints are $P_{a}$ and $P_{b}$. Observe that no triangular face is shared by more than two tetrahedra.

used to obtain a univariate closure polynomial. Here we solve this problem departing from this standard approach. The proposed method can be summarized as follows. The distance between the strip endpoints is first derived by iterating a basic operation involving only two neighboring tetrahedra over the whole strip. This leads to a scalar equation containing radical terms. We will see how clearing these radicals is a trivial task, and how the resulting polynomial contains, in general, factor terms that correspond to singularities of the formulation that depend on the chosen variable length. Since these terms can be easily spotted beforehand, their elimination is just a matter of iterative polynomial division until a no null remainder is obtained. The result is the sought-after univariate closure polynomial obtained without variable eliminations or trigonometric substitutions.

Next, we detail this procedure and then we apply it to derive the minimal degree closure polynomial for two widely studied parallel platforms: the decoupled parallel platform, and a 4-4 platform with planar base and moving platform.

\section{Obtaining the Closure Polynomials}

Given a set of points, the valid distances between them can be characterized using the theory of Cayley-Menger determinants $[1,6,8]$. The Cayley-Menger bideterminant of the two sets of points $P_{i_{1}}, \ldots, P_{i_{n}}$ and $P_{j_{1}}, \ldots, P_{j_{n}}$ is defined as

$$
D\left(i_{1}, \ldots, i_{n} ; j_{1}, \ldots, j_{n}\right)=2\left(-\frac{1}{2}\right)^{n}\left|\begin{array}{cccc}
0 & 1 & \ldots & 1 \\
1 & s_{i_{1}, j_{1}} & \ldots & s_{i_{1}, j_{n}} \\
1 & \vdots & \ddots & \vdots \\
1 & s_{i_{n}, j_{1}} & \ldots & s_{i_{n}, j_{n}}
\end{array}\right|,
$$


where $s_{i, j}$ stands for the squared distance between $P_{i}$ and $P_{j}$.

If the two sets of points are the same, then $D\left(i_{1}, \ldots, i_{n}\right)=D\left(i_{1}, \ldots, i_{n} ; i_{1}, \ldots, i_{n}\right)$ is called the Cayley-Menger determinant of the involved set of points. The CayleyMenger determinant $D\left(i_{1}, \ldots, i_{n}\right)$ is proportional to the squared volume of the simplex spanned by $P_{i_{1}}, \ldots, P_{i_{n}}$ in $\mathbb{R}^{n-1}$.
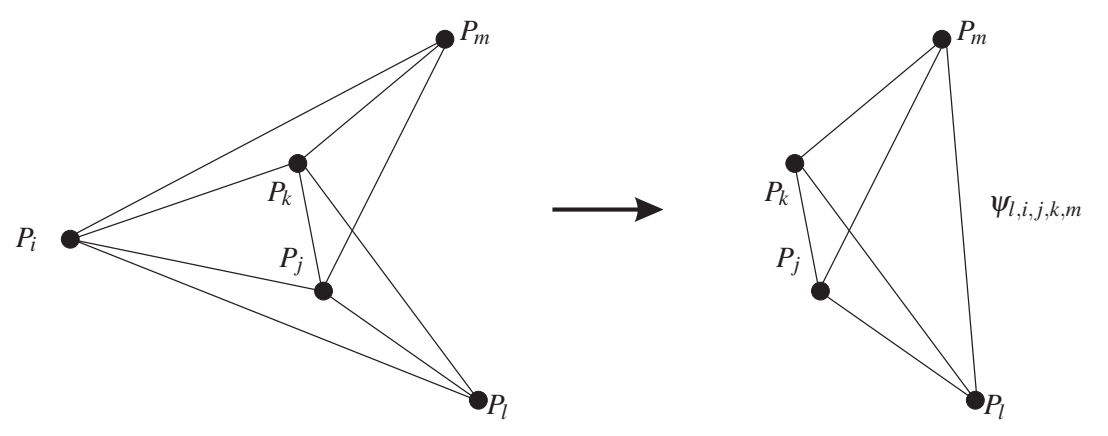

Fig. 2 Substitution rule.

Now, let us suppose the two neighboring tetrahedra in Fig. 2-left belong to a strip of tetrahedra in $\mathbb{R}^{3}$. The squared distance between $P_{l}$ and $P_{m}$ can be expressed as (see [7] for details):

$$
s_{l, m}=\frac{2}{D(i, j, k)}\left(\left.D(i, j, k, l ; i, j, k, m)\right|_{s_{l, m}=0} \pm \sqrt{D(i, j, k, l) D(i, j, k, m)}\right) .
$$

where the \pm sign accounts for the two possible solutions depending on the relative orientation between the two tetrahedra. To lighten the notation, (2) will be simply written as $s_{l, m}=\Psi_{l, i, j, k, m}$. If some of the distances involved in $\Psi_{l, i, j, k, m}$ are taken as variables, they will be made explicit in parenthesis. For example, if $s_{i, j}$ and $s_{i, k}$ are variables, we will write $s_{l, m}=\Psi_{l, i, j, k, m}\left(s_{i, j}, s_{i, k}\right)$.

If one of the points in the set $\left\{P_{i}, P_{j}, P_{k}\right\}$ does not belong to any other tetrahedron in the strip, it can be removed from the strip provided that a rod connecting $P_{l}$ and $P_{m}$ is introduced with the double-valued length given by (2) [Fig. 2-right]. This reduces the number of tetrahedra in the strip by one. Then, by repeating this operation until the strip contains only two tetrahedra, the distance between the tetrahedral strip endpoints is finally obtained as a $2^{n-2}$-valued function, where $n$ is the number of tetrahedra in the strip.

To obtain the closure condition as a polynomial in terms of a given rod length, the first step consists in taking the numerator of the rational form of the obtained function and then clearing radicals. As radicals will appear nested, they are cleared using an iterative process starting from the outer one. At each step of this process, the expressions involving a radical will have the general form 


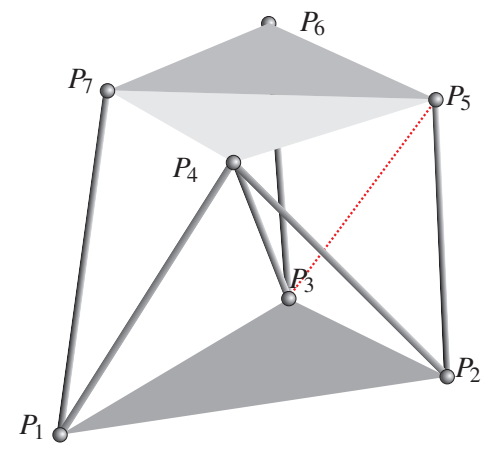

$\mathbf{S}=\left(\begin{array}{ccccccc}0 & 34 & 49 & 62 & ? & ? & 108 \\ 34 & 0 & 41 & 58 & 108 & ? & ? \\ 49 & 41 & 0 & 68 & ? & 126 & ? \\ 62 & 58 & 68 & 0 & 38 & 91 & 34 \\ ? & 108 & ? & 38 & 0 & 85 & 74 \\ ? & ? & 126 & 91 & 85 & 0 & 197 \\ 108 & ? & ? & 34 & 74 & 197 & 0\end{array}\right)$

Fig. 3 Decoupled parallel manipulator, with non-planar moving platform, used as example.

$$
\alpha_{0}+\alpha_{1} \sqrt{r}+\alpha_{2}(\sqrt{r})^{2}+\alpha_{3}(\sqrt{r})^{3}+\cdots=0,
$$

which can be rewritten as

$$
\left(\alpha_{0}+\alpha_{2} r+\alpha_{4} r^{2}+\ldots\right)+\sqrt{r}\left(\alpha_{1}+\alpha_{3} r+\alpha_{5} r^{2}+\ldots\right)=0
$$

This equation can be unfolded into two equations, one for each sign of $\sqrt{r}$. Since we are interested in the roots of both equations, we obtain their product, which can be written as

$$
\left(\alpha_{0}+\alpha_{2} r+\alpha_{4} r^{2}+\ldots\right)^{2}-r\left(\alpha_{1}+\alpha_{3} r+\alpha_{5} r^{2}+\ldots\right)^{2}=0
$$

While clearing radicals as explained above introduces no extraneous roots, one cannot expect for the obtained polynomial to be of minimal degree. This is due to the presence of singularities of the formulation. Indeed, let us suppose that the closure polynomial is expressed in terms of the squared rod length $s_{i, j}$. If a rod with variable length belongs to a shared face, this face degenerates for some values of $s_{i, j}$. When this happens, the three points defining the face get aligned and the tetrahedral strip can be decomposed into two parts so that one can freely rotate with respect the other about the axis defined by these three aligned points. As we will see, terms corresponding to these degenerate configurations will appear in the closure polynomial. They can be easily removed by iteratively dividing the closure polynomial by them until the remainder is not null.

\section{Examples}

Next, we apply the technique explained above to solve the foward kinematics of a decoupled platform and a 4-4 platform with planar base and platform (see Fig. 3 and Fig. 5, respectively). The decoupled platform owes its name to the fact that three legs permit the rotation of the platform about a point whose location is controlled 
by the other three. Since the forward kinematics for the translational part is trivial, the interest of this linkage lies in the spherical part for which a minimal closing polynomial of degree 8 on a squared variable was first derived in [2]. In [7], this derivation is simplified by using the closure polynomial of the so-called double banana. Despite the simpler derivation, variable eliminations were still necessary. For the chosen 4-4 platform with planar base and platform, a minimal 12th-degree closure polynomial was first derived in [3]. The derivation was far from trivial and applicable only to this particular platform. To properly compare our results with those reported in [7] and [3], we use the same numerical examples.

First, let us consider the decoupled platform defined by the squared distance matrix $\mathbf{S}$ appearing in Fig. 3, where $s_{i, j}=\mathbf{S}(i, j)$. It can be topologically described as the strip of tetrahedra shown in Fig. 4-left. Applying the substitution presented in the previous section three times (see Fig. 4), we have

$$
\begin{aligned}
& s_{3,7}=\Psi_{3,4,5,6,7}\left(s_{3,5}\right), \\
& s_{2,7}=\Psi_{2,3,4,5,7}\left(s_{3,7}, s_{3,5}\right), \\
& s_{1,7}=\Psi_{1,2,3,4,7}\left(s_{2,7}, s_{3,7}\right) .
\end{aligned}
$$

The numerator of the rational form resulting from substituting (6) in (7), and the result in (8), can be written as:

$$
\begin{aligned}
R_{1} & -1346.0 R_{2}+7899650 R_{3}+24942632734 s_{3,5}+1402 R_{3} s_{3,5}{ }^{2} \\
& -323070338 s_{3,5}{ }^{2}+741658 s_{3,5}{ }^{3}-208500 R_{3} s_{3,5}+528767086008=0,
\end{aligned}
$$

where

$$
\begin{aligned}
& R_{1}=\sqrt{-2027718 R_{2}^{2}+4695768 R_{2} R_{3} s_{3,5}^{2}-729124704 R_{2} R_{3} s_{3,5}+\ldots}, \\
& R_{2}=\sqrt{100464 R_{3}^{2} s_{3,5}^{2}-19847712 R_{3}^{2} s_{3,5}+115799664 R_{3}^{2}-\ldots}, \\
& R_{3}=\sqrt{-3481450 s_{3,5}^{2}+806976100 s_{3,5}-27440188650}
\end{aligned}
$$

The full expressions for $R_{1}$ and $R_{2}$ are not included here due to space limitations.

Now, clearing the radicals as described in Section 2, we obtain a polynomial of 24th-degree. It is not of minimal degree because the rod connecting $P_{3}$ and $P_{5}$ belongs to the shared face defined by $P_{3}, P_{4}$, and $P_{5}$ which is singular when $D(3,4,5)=0$, that is, when $s_{3,5}^{2}-214 s_{3,5}+961=0$. By iteratively dividing the obtained polynomial by this singular factor until the remainder is not null, we get

$$
\begin{aligned}
s_{3,5}^{16} & -1.6652 \cdot 10^{4} s_{3,5}^{15}+1.2722 \cdot 10^{6} s_{3,5}^{14}-5.8952 \cdot 10^{8} s_{3,5}^{13}+1.8487 \cdot 10^{11} s_{3,5}^{12} \\
& -4.1525 \cdot 10^{13} s_{3,5}^{11}+6.9146 \cdot 10^{15} s_{3,5}^{10}-8.7384 \cdot 10^{17} s_{3,5}^{9}+8.5338 \cdot 10^{19} s_{3,5}^{8} \\
& -6.5533 \cdot 10^{21} s_{3,5}^{7}+4.0715 \cdot 10^{23} s_{3,5}^{6}-2.1848 \cdot 10^{25} s_{3,5}^{5}+1.1165 \cdot 10^{27} s_{3,5}^{4} \\
& -5.4256 \cdot 10^{28} s_{3,5}^{3}+2.0923 \cdot 10^{30} s_{3,5}^{2}-5.0066 \cdot 10^{31} s_{3,5}+5.2479 \cdot 10^{32},
\end{aligned}
$$




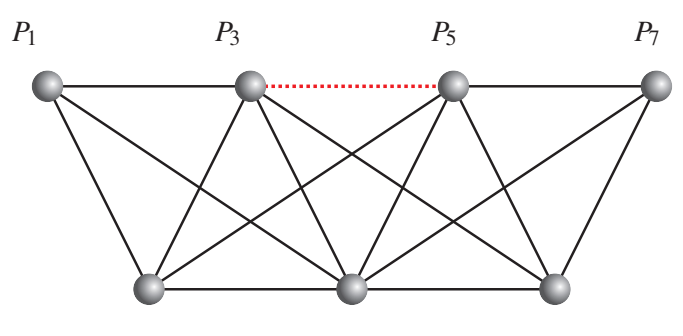

$P_{2} \quad P_{4} \quad P_{6}$

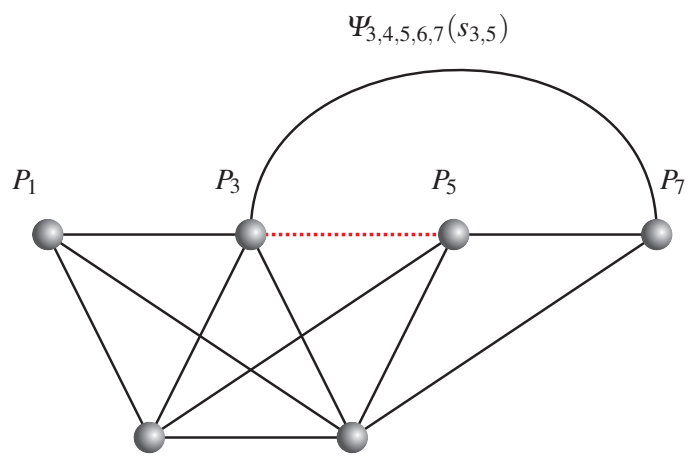

$P_{2} \quad P_{4}$

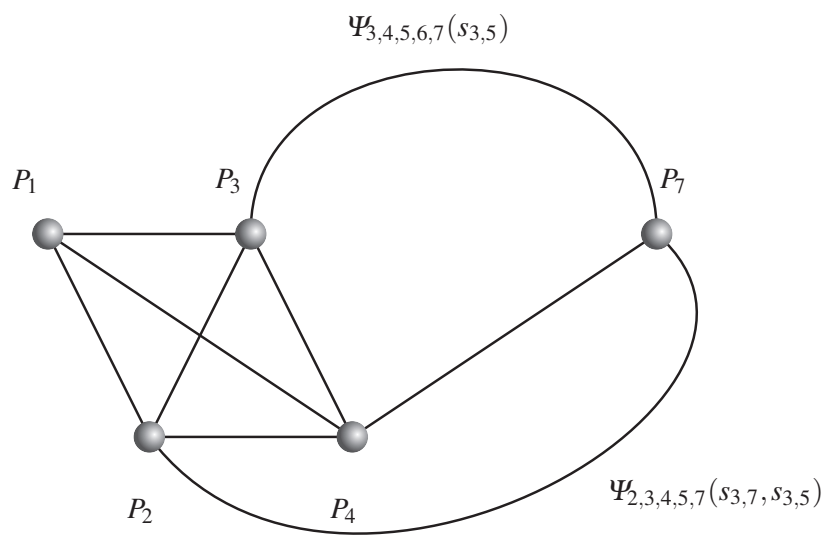

Fig. 4 The decoupled parallel platform in Fig. 3 can be topologically described as the strip of four tetrahedra in which the distance between $P_{3}$ and $P_{5}$ is variable and the distance between its endpoints, $P_{1}$ and $P_{7}$, is known. The application of the substitution rule presented in Section 2 to this strip (left) permits to sequentially eliminate $P_{6}$ (center) and $P_{5}$ (right). 


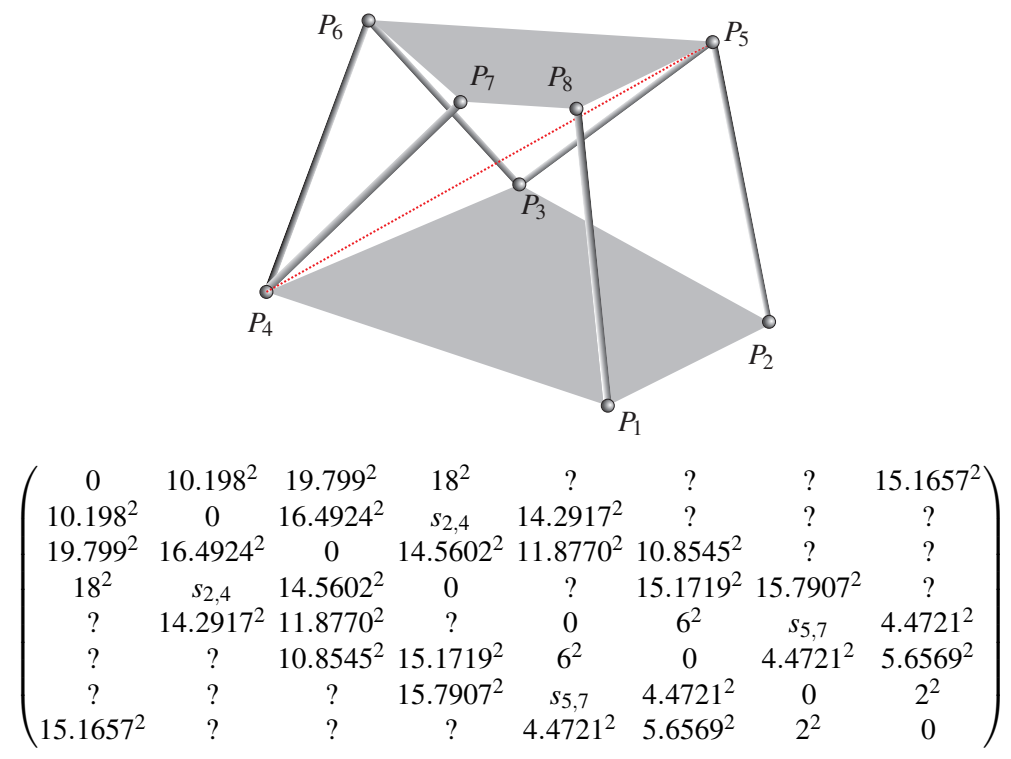

Fig. 5 4-4 parallel manipulator used as example. Since the base and the moving platform are convex planar quadrilaterals, $s_{2,4}$ and $s_{5,7}$ are unambiguously determined by the other known distances.

which coincides with the closure polynomial reported in [7], but obtained in a much simpler way.

As a second example, let us consider the 4-4 parallel platform appearing in Fig. 5. Its forward kinematics is known to have 24 solutions [3]. However, they can be split in two sets that are symmetric with respect to the base. Since the distance-based formulation is invariant to this symmetry, we will get a 12th-degree closure polynomial. The two sets of configurations are obtained in the coordinatization process using trilateration $[4,5,8]$.

Applying the substitution presented in the previous section four times (see Fig. 6), we have that

$$
\begin{array}{ll}
s_{4,8}=\Psi_{4,5,6,7,8}\left(s_{4,5}\right), & s_{2,8}=\Psi_{2,3,4,5,8}\left(s_{3,8}, s_{4,8}\right), \\
s_{3,8}=\Psi_{3,4,5,6,8}\left(s_{4,8}, s_{4,5}\right), & s_{1,8}=\Psi_{1,2,3,4,8}\left(s_{2,8}, s_{3,8}, s_{4,8}\right) .
\end{array}
$$

After a proper sequence of forward substitutions in the above four equations, $s_{1,8}$ can be expressed only in terms of $s_{4,5}$. Since this parallel platform has planar base and platform, $\Psi_{4,5,6,7,8}$ and $\Psi_{1,2,3,4,8}$ are single-valued functions. Only $\Psi_{3,4,5,6,8}$ and $\Psi_{2,3,4,5,8}$ contribute with square roots to the obtained closure condition. Eliminating them as explained leads to a 52nd-degree polynomial in $s_{4,5}$. In this case, the rod connecting $P_{4}$ and $P_{5}$ belongs to two shared faces (the ones defined by $P_{4} P_{5} P_{6}$ and $\left.P_{3} P_{4} P_{5}\right)$, whose associated singular terms are $s_{4,5}^{2}-706.1251 s_{4,5}+5031.9580$, and $s_{4,5}{ }^{2}-532.3731 s_{4,5}+37708.4160$. After iteratively dividing the obtained polyno- 

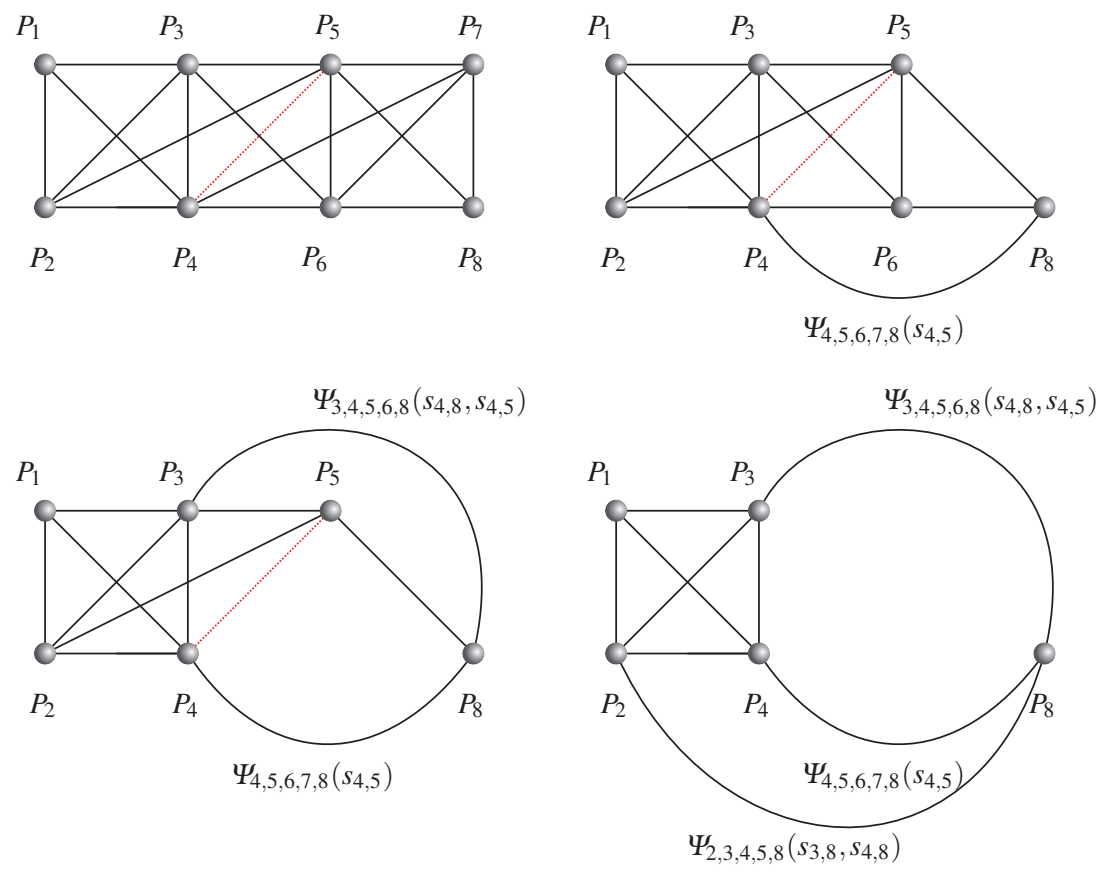

Fig. 6 The 4-4 parallel manipulator in Fig. 5 can be topologically described as the strip of five tetrahedra in which the distance between $P_{4}$ and $P_{5}$ is variable and the distance between its endpoints, $P_{1}$ and $P_{8}$, is known. The application of the substitution rule to this strip (top left) permits to sequentially eliminate $P_{7}$ (top right), $P_{6}$ (bottom left), and $P_{5}$ (bottom right).

mial by these two factors until the remainder is not null, the following 12th-degree polynomial is obtained

$$
\begin{aligned}
s_{4,5}^{12} & -0.676 \cdot 10^{3} s_{4,5}^{11}-3.873 \cdot 10^{6} s_{4,5}^{10}+5.400 \cdot 10^{9} s_{4,5}^{9}-9.858 \cdot 10^{12} s_{4,5}^{8} \\
& -2.327 \cdot 10^{15} s_{4,5}^{7}+1.967 \cdot 10^{18} s_{4,5}^{6}-7.316 \cdot 10^{20} s_{4,5}^{5}+1.518 \cdot 10^{23} s_{4,5}^{4} \\
& -1.834 \cdot 10^{25} s_{4,5}^{3}+1.257 \cdot 10^{27} s_{4,5}^{2}-4.432 \cdot 10^{28} \mathrm{~S} 4_{5}+6.171 \cdot 10^{29} .
\end{aligned}
$$

This polynomial has six roots that lead to real configurations of the moving platform obtained by coordinatization via trilaterations $[5,8]$. These roots and the corresponding configurations appear in Fig. 7. They coincide with the solutions reported in [3] obtained using an ad hoc intricate method.

\section{Conclusions}

It has been explained how to obtain closure polynomials for tetrahedral strips in terms of the involved rod lengths and the distance between the strip endpoints, and 

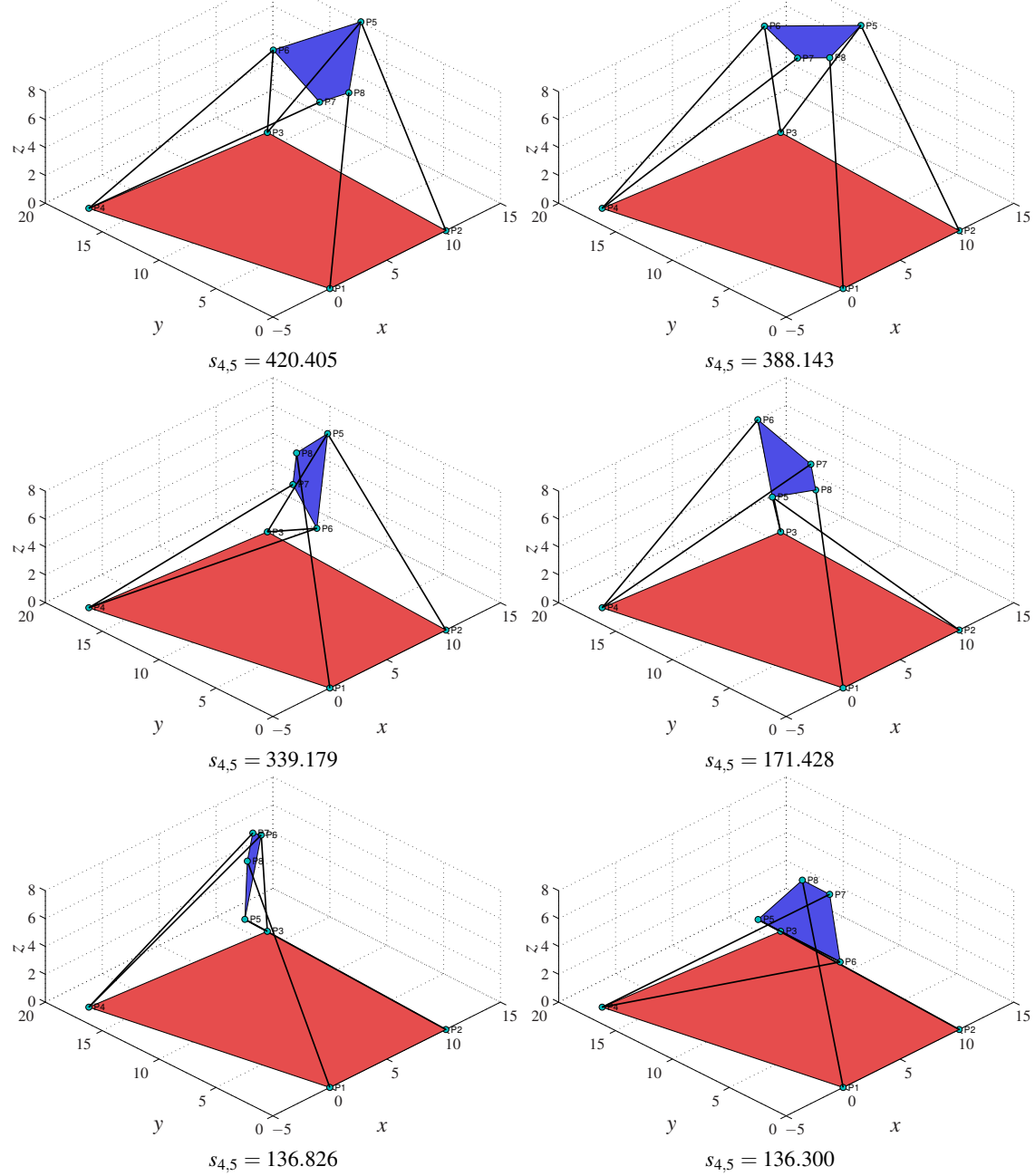

Fig. 7 Forward kinematics solutions of the 4-4 manipulator used as example. The mirror configurations with respect to the base are also solutions, but they are not represented.

how this technique can be applied to solve some position analysis problems. However, this technique cannot incorporate orientation constraints between tetrahedra at different parts of the strip. As a consequence, if applied to a case in which such constraints are necessary, the obtained closure polynomial would not be of minimaldegree as some of its roots would violate these constraints. Despite this important limitation, it supersedes the method presented in [7] in scope and simplicity, thus providing a better starting point for a complete generalization to three dimensions 
of the techniques developed for the position analysis of planar linkages using Distance Geometry.

Acknowledgements This work has been partially supported by by the Spanish Ministry of Economy and Competitiveness under project DPI2014-57220-C2-2-P.

\section{References}

1. Havel, T.: Some examples of the use of distances as coordinates for Euclidean geometry. Journal of Symbolic Computation 11(5-6), 579-593 (1991)

2. Innocenti, C., Parenti-Castelli, V.: Echelon form solution of direct kinematics for the general fully-parallel spherical wrist. Mechanism and Machine Theory 28, 553-561 (1993)

3. Lin, W., Griffis, M., Duffy, J.: Forward displacement analyses of the 4-4 Stewart platforms. ASME Journal of Mechanical Design 114(3), 444-450 (1992)

4. Porta, J.M., Ros, L., Thomas, F.: Inverse kinematics by distance matrix completion. In: International Workshop on Computational Kinematics (2005)

5. Porta, J.M., Ros, L., Thomas, F.: On the trilaterable six-degree-of-freedom parallel and serial manipulators. In: IEEE International Conference on Robotics and Automation, pp. 960-967 (2005)

6. Porta, J.M., Ros, L., Thomas, F., Torras, C.: A branch-and-prune algorithm for solving systems of distance constraints. In: IEEE International Conference on Robotics and Automation, pp. 342-348 (2003)

7. Rojas, N., Thomas, F.: The closure condition of the double banana and its application to robot position analysis. In: International Conference on Robotics and Automation, pp. 4641-4646 (2013)

8. Thomas, F., Ros, L.: Revisiting trilateration for robot localization. IEEE Transactions on Robotics 21(1), 93-101 (2005) 